\title{
Diagnosis and Management of Gastric Intestinal Metaplasia: Current Status and Future Directions
}

\author{
Robert J. Huang ${ }^{1}$, Alyssa Y. Choi ${ }^{2}$, Camtu D. Truong ${ }^{3}$, Matthew M. Yeh ${ }^{3}$, and Joo Ha Hwang ${ }^{1}$ \\ ${ }^{1}$ Division of Gastroenterology and Hepatology, Stanford University, Stanford, CA, and Departments of ${ }^{2}$ Medicine and ${ }^{3}$ Pathology, University of \\ Washington, Seattle, WA, USA
}

\begin{abstract}
Gastric intestinal metaplasia (GIM) is a known premalignant condition of the human stomach along the pathway to gastric cancer (GC). Histologically, GIM represents the replacement of normal gastric mucosa by mucin-secreting intestinal mucosa. Helicobacter pylori infection is the most common etiologic agent of GIM development worldwide. The prevalence of GIM is heterogeneous among different regions of the world and correlates with the population endemicity of $H$. pylori carriage, among other environmental factors. GC remains the third leading cause of cancer-related mortality globally. GIM is usually diagnosed by upper endoscopy with biopsy, and histologic scoring systems have been developed to riskstratify patients at highest risk for progression to GC. Several recent endoscopic imaging modalities may improve the optical detection of GIM and early GC. Appropriate surveillance of GIM may be cost effective and represents an opportunity for the early diagnosis and therapy of GC. Certain East Asian nations have established population-level programs for the screening and surveillance of GIM; guidelines regarding GIM surveillance have also recently been published in Europe. By contrast, few data exist regarding the appropriateness of surveillance of GIM in the United States. In this review, we discuss the pathogenesis, epidemiology, diagnosis, and management of GIM with an emphasis on the role of appropriate endoscopic surveillance. (Gut Liver 2019;13:596603)
\end{abstract}

Key Words: Helicobacter pylori; Epidemiology; Stomach

\section{INTRODUCTION}

Gastric intestinal metaplasia (GIM) is defined as the replacement of the oxyntic or antral gastric mucosa by intestinal mu- cosa consisting of Paneth, goblet, and absorptive cells. ${ }^{1}$ GIM is an important precursor lesion in the pathway to gastric cancer (GC), ${ }^{2-5}$ and regional prevalence of GIM correlates closely with incidence of GC worldwide. ${ }^{6}$ History of Helicobacter pylori infection, race and ethnicity, immigration status, age, family history, and other environmental factors may all mediate both the risk for GIM, and the risk of progression to GC. ${ }^{7-10}$

In Western Europe, it has been estimated that annual rates of progression onto GC to be approximately 0.1\%, 0.25\%, 0.6\%, and 6\% for patients with atrophic gastritis (AG), GIM, mildto-moderate dysplasia, and severe dysplasia, respectively. ${ }^{10}$ In contrast, in East Asia it has been estimated that annually 1.8\%, $10 \%$, and $73 \%$ of patients with AG, GIM, and dysplasia will progress to GC, respectively, highlighting the importance of geography and ethnicity/race in determining risk for progression. ${ }^{11}$ While GC rates have declined worldwide (including in East Asia) due in part to improvements in sanitation and recognition of the carcinogenic role of $H$. pylori infection, GC remains the third most common cause of cancer-related mortality worldwide and results in over 700,000 deaths annually. ${ }^{11}$ Thus, a more complete understanding of the pathobiology GIM, the appropriate surveillance of patients with GIM, and the changing therapeutic options for patients diagnosed with early GC may all lead to improvement in GC-related morbidity and mortality.

\section{PATHOGENESIS OF GIM}

Correa's cascade is a widely accepted model of the pathogenesis of GC (Fig. 1). ${ }^{12}$ The first step in this cascade is the development of chronic mucosal inflammation, mediated through both polymorphonuclear cells and mononuclear cells, which can occur as a consequence of infection with $H$. pylori or through other environmental insults or through autoimmunity (in the

Correspondence to: Joo Ha Hwang

Division of Gastroenterology and Hepatology, Stanford University, 300 Pasteur Dr. H0268, MC:5244, Stanford, CA 94305, USA

Tel: +1-650-497-6313, Fax: +1-650-498-6323, E-mail: jooha@stanford.edu

Received on May 20, 2019. Revised on July 8, 2019. Accepted on July 8, 2019. Published online August 12, 2019. pISSN 1976-2283 eISSN 2005-1212 https://doi.org/10.5009/gnl19181

(a) This is an Open Access article distributed under the terms of the Creative Commons Attribution Non-Commercial License (http://creativecommons.org/licenses/by-nc/4.0) which permits unrestricted non-commercial use, distribution, and reproduction in any medium, provided the original work is properly cited. 


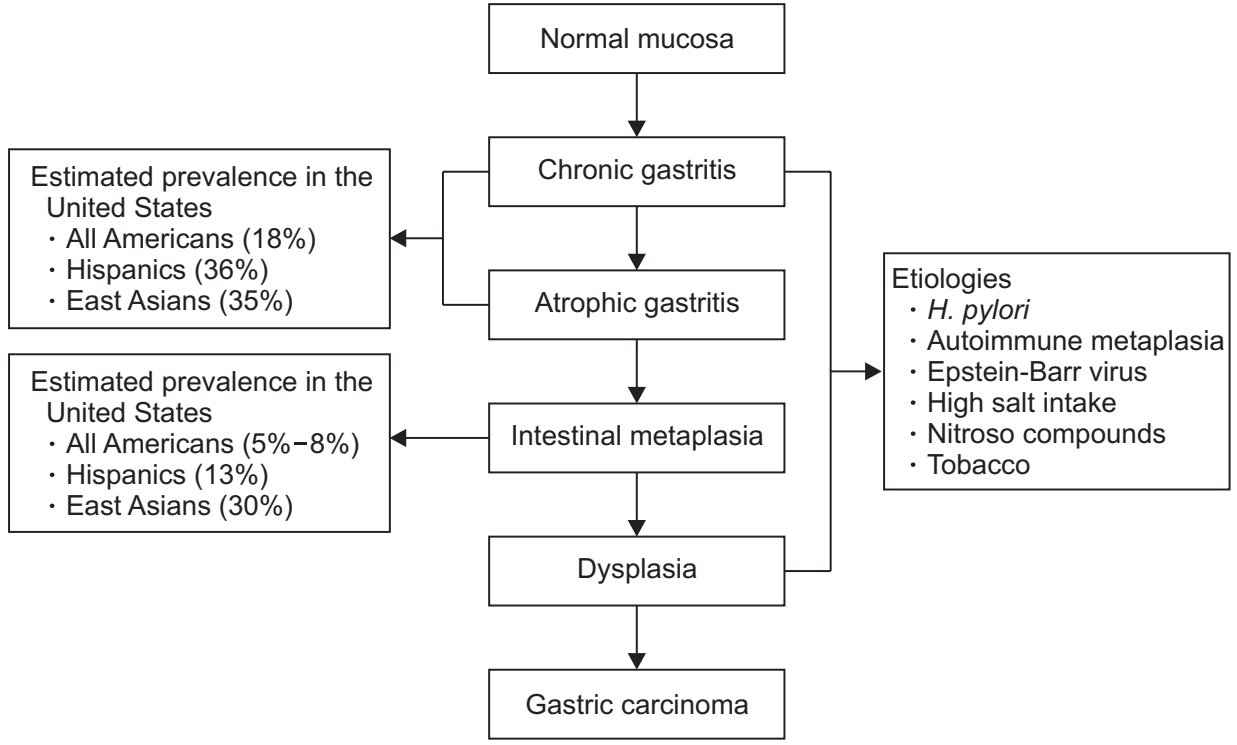

Fig. 1. Correa's cascade, a model for the histologic progression towards gastric cancer. Infection with $\mathrm{He}-$ licobacter pylori is the single most common etiologic environmental factor that precipitates the cascade. case of autoimmune metaplastic AG). Due to poorly understood mechanisms, chronic mucosal inflammation leads eventually to multifocal glandular atrophy, loss of parietal cell mass, and loss of acidic secretory function. As atrophy progresses, replacement with intestinal-type epithelium characterized most prominently by the presence of mucin-containing goblet cells occurs (Fig. 2). As atrophy and metaplasia to intestinal-type mucosa progresses, generally in an antrum to corpus pattern in $H$. pylori-induced gastritis, stomach acidity and production of pepsinogen I decreases. Dysplasia is the next step in the cascade, characterized by a neoplastic cellular phenotype with large, hyperchromatic cells and disorganized nuclei. While dysplastic cells have a neoplastic phenotype, they continue to respect cellular boundaries and lack penetration across the lamina propria, which is the defining feature of invasive carcinoma. Notably, there are regional differences between Western and Japanese pathologists regarding nomenclature of dysplasia and early mucosa carcinoma, discussed in detail below.

\section{ENVIRONMENT RISK FACTORS}

\section{Helicobacter pylori}

H. pylori is a Gram-negative bacterium, and worldwide the most important etiologic agent for the development of both AG and GIM; it has been classified by the World Health Organization as a type I carcinogen. ${ }^{13}$ It colonizes the gastric epithelium of more than 50\% of adults worldwide, with prevalence rates ranging from $30 \%$ in industrialized areas to $90 \%$ in developing countries and Eastern Asia. ${ }^{14}$ A history of $H$. pylori infection is associated with a 3-fold increase in lifetime odds of developing non-cardia GC, and H. pylori is believed to be responsible for $75 \%$ to $95 \%$ of all GC cases. ${ }^{15,16}$

H. pylori may mediate metaplasia and dysplasia through numerous putative mechanisms. Infection activates immune

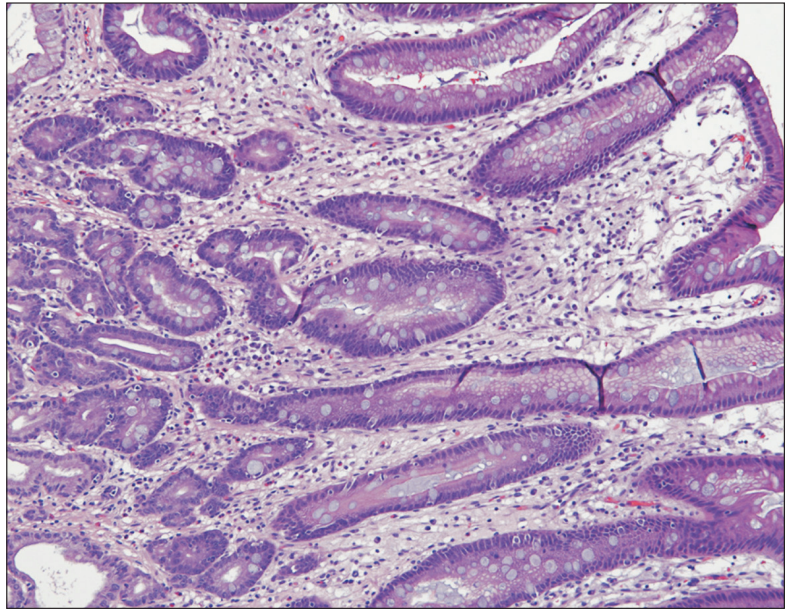

Fig. 2. High-power view $(H \& E, \times 100)$ of intestinal metaplasia showing metaplastic goblet cells on the surface and in the foveolar epithelium.

cells, including macrophages, T cells and B cells, promoting the release of pro-inflammatory cytokines and chronic inflammation which may induce DNA methylation and reactive oxygen species. ${ }^{17,18}$ The genes susceptible to epigenetic changes induced by $H$. pylori include genes linked to intercellular junctions (Ecadherin, Vezatin, Connexin32, and Connexin43), cell cycle regulation (CDKN2A), DNA repair (hMHL-1), inflammation (TFF-2, COX-2), transcription (RUNX3, FOXD3, USF1 and USF2, GATA4 , and GATA-5), and tumor suppression (LOX and HRASLS). ${ }^{19}$

\section{Eradication of $\boldsymbol{H}$. pylori}

Eradication reduces inflammation associated with $H$. pylori and restores acid secretion, which may regulate bacterial growth. Yet population-level screening and eradication of $H$. pylori as a method to reduce the risk of GC remains contro- 
versial. In a recent systematic review and meta-analysis of six trials enrolling 6,497 patients, the relative risk of developing GC among treated patients was 0.66 compared to untreated patients; however, treatment did not appear to have a protective effect against either GC-related or all-cause mortality (due in part to insufficient sample size), and the number needed to treat was highly variable based upon gender and ethnicity. ${ }^{20,21}$ If $H$. pylori screening is to occur, targeting younger individuals (in whom the preneoplastic changes along Correa's cascade have yet to develop) appear to be the most cost-effective strategy. ${ }^{22,23}$

\section{Other environmental risk factors}

Individuals with a positive family history, particularly firstdegree relatives with GC, carry an increased risk for premalignant gastric lesions and GC. However, it is estimated that less than $10 \%$ of GCs are hereditary, with the remainder being sporadic. $^{24}$ The combination of a virulent bacterial strain, a genetically susceptible host, and a predisposed gastric environment may be required for cancer to develop. ${ }^{25}$ In a systematic review and meta-analysis of 12 cohort and 30 case-control studies, high red-meat and processed-meat intake were both associated with an approximately 50\% increase in relative risk for subsequent development of GC. ${ }^{26}$ Prior to widespread availability of electric refrigeration, salting and pickling was a predominant method of food preservation, especially in East Asia. High salt intake has associated with risk for GC, whereas years of refrigeration availability has associated against risk for GC in a casecontrol study. ${ }^{27}$ Tobacco smoking significantly increases risk for GC, with an observed dose-dependent effect. ${ }^{28}$ Notably, tobacco cessation seems to mitigate some of this risk. ${ }^{29}$ Obesity also appears to be an independent risk factor for the development of GC. $^{30,31}$

\section{HISTOLOGIC DIAGNOSIS AND RISK STRATIFICATION}

\section{The Sydney System}

The Updated Sydney System is a comprehensive endoscopic and histologic sampling protocol by which to stage $H$. pylori, AG, and GIM. ${ }^{32}$ Biopsy sites are standardized (Fig. 3), with two biopsies taken from the antrum (along the lesser and greater curvatures), one biopsy taken from the incisura angularis, and two biopsies taken from the stomach body (along the lesser and greater curvatures). Histologic scoring of $H$. pylori, AG, and GIM occurs on a visual-analogue scale, with values of none, mild, moderate and marked.

As a corollary to the Sydney System, the Operative Link for Gastritis Assessment (OLGA) was proposed as a way to stage gastric atrophy. In this system, the stage of AG is determined by combining the degree of atrophy with the location of the biopsy. The degree of atrophy is scored on a scale of 0 to 3 (none, mild, moderate), or marked by combining the overall antrum score with the overall corpus score, the stage (0 to IV) is determined..$^{33}$
Retrospective studies have shown that gastric adenocarcinoma tends to develop in patients with OLGA stage III or IV while it rarely or never develops in those with stage 0, I or II. ${ }^{33,34}$

Similar to OLGA, the Operative Link for Gastric Intestinal Metaplasia (OLGIM) is a scoring system based upon the Sydney System to risk-stratify patients at highest risk for progressing from GIM to GC, with the aim of reducing interobserver variability due to GIM being more readily recognized and quantifiable compared to AG. ${ }^{35}$ It adopts an ordinal scoring system similar to OLGA, with each individual biopsy scored according to a visual-analogue scale, and reports an overall stage. Additional studies are needed to compare the diagnostic and testing characteristics of OLGIM.

\section{Mucin-based classification}

A separate histologic classification system based on morphologic features combined with mucin expressed patterns has been proposed..$^{36}$ In this classification scheme, type I (complete) GIM is characterized by the presence of goblet cells secreting sialomucins, columnar and/or Paneth cells. Type II (incomplete) also contains goblet cells, but lacks columnar and/or Paneth cells. Type III (or IIa) also demonstrate the presence of goblet cells and absence of columnar and/or Paneth cells, but sulfomucins rather than sialomucins are the predominantly expressed mucins. The relative risk of developing cancer has been observed to be higher in type III intestinal metaplasia. ${ }^{37}$

A1: $3 \mathrm{~cm}$ from pylorus, along lesser curve A2: $3 \mathrm{~cm}$ from pylorus, along greater curve IA: Incisura angularis

$B 1:$ Body lesser curve, $4 \mathrm{~cm}$ proximal to IA

$\mathrm{B} 2$ : Body greater curve, $8 \mathrm{~cm}$ distal to cardia

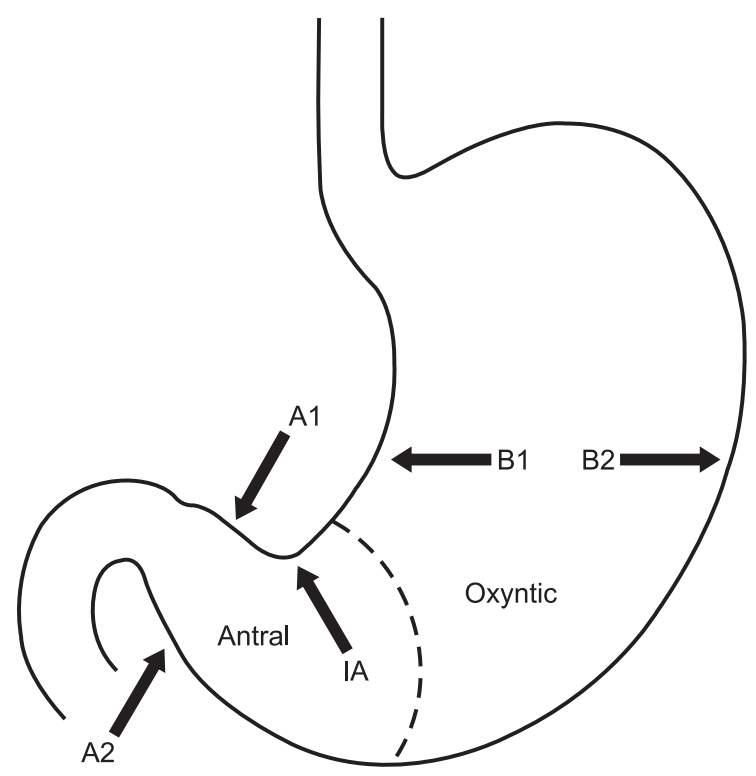

Fig. 3. Updated the Sydney System biopsy protocol, with two biopsies taken from the antrum, one biopsy taken from the incisura, one biopsy taken along the lesser curvature of the gastric body, and one biopsy taken along the greater curvature of the gastric body. 
Utilizing immunohistochemistry to differentiate between gastric mucins and intestinal mucins has also been explored. ${ }^{38}$ Weak expression of MUC2 (intestinal mucin) and the absence of MUC1 (epithelial membrane mucin), MUC5AC (gastric mucin), and MUC6 (gastric mucin), characterize the complete type of intestinal metaplasia. Incomplete intestinal metaplasia is characterized by strong expression of MUC1 (epithelial membrane mucin), MUC2 (intestinal mucin), and MUC5AC (gastric mucin). Das-1 (a marker of colonic epithelium) has been seen to be expressed in high-risk, incomplete phenotypes. ${ }^{39}$ It has also been reported that the complete type of intestinal metaplasia expresses sucrase, which is specific to absorptive cells of the small intestine, much more frequently than the incomplete type of intestinal metaplasia. ${ }^{40}$

From these observations, it has been hypothesized that complete intestinal metaplasia displays predominantly small intestinal phenotypic markers such as MUC2 and sucrase, while incomplete intestinal metaplasia expresses gastric phenotypic markers MUC5AC and large intestinal phenotypic markers such as Das-1.

\section{Dysplasia and carcinoma}

There exist regional differences in definition between Western and Japanese pathologists in the classification of dysplastic lesions. ${ }^{41}$ The term dysplasia is used by Western pathologists when there is no evidence of an invasive component, whereas carcinoma is defined as lesions exhibiting invasion into or beyond the lamina propria. In contrast, the diagnosis of "mucosal carcinoma" is rendered by Japanese pathologists when there is sufficient cytoarchitectural complexity regardless whether there is invasion into the lamina propria. Thus, many lesions that are diagnosed as high-grade dysplasia by most Western pathologists are diagnosed as carcinoma by most Japanese pathologists. These differences may be mostly semantic, as most Western clinicians would refer patients with high-grade dysplasia to either endoscopic or surgical resection, similar to the management of early mucosal carcinoma in Japan or elsewhere in East Asia.

Both architectural and cytologic features are important in evaluating for low-grade and high-grade dysplasia. ${ }^{41}$ In lowgrade dysplasia, architectural changes are relatively mild and characterized by glandular crowding and disarray, mild glandular branching and rare glandular budding. The nuclei display hyperchromasia and elongation, mild to moderate mitotic activity, are still basally located, and maintain nuclear polarity. Highgrade dysplasia demonstrates more complex architecture with marked glandular crowding and disarray, back-to-back glands with intraluminal folds and cribiforming. Glandular branching and budding are frequently seen. The cytologic atypia is more severe with markedly increased mitotic activity and presence of atypical mitoses. The nuclei usually reach the luminal surface of the cell cytoplasm, displaying loss of nuclear polarity and appear round and vesicular. In invasive carcinoma, infiltration of the lamina propria by either glands or single cells is seen.

\section{ENDOSCOPIC IMAGING MODALITIES}

Conventional white light endoscopy cannot accurately differentiate between and diagnose preneoplastic gastric lesions. ${ }^{42}$ Therefore, research has been directed at endoscopic techniques to improve diagnosis of preneoplastic gastric lesions and assess the invasiveness of cancerous lesions. ${ }^{43}$ While these methods are more sensitive in detecting GIM and early GC, they may be limited by significant interobserver variability. ${ }^{43,44}$

\section{Narrow-band imaging}

Narrow-band imaging (NBI) is an endoscopic technology relying on the filtering of white light into defined wave lengths to maximize absorption by hemoglobin, and limit penetration of light beyond the mucosal surface. Given its shorter wavelength, blue light penetrates less deeply than red light and thus enhances the imaging of fine structures of the mucosal surface without the use of dyes. On magnified NBI, normal stomach mucosa should demonstrate a regular circular pattern, homogeneously spaced gastric pits (Fig. 4). ${ }^{45,46}$ GIM is characterized on NBI by the presence of features including tubule-villous mucosal pattern, irregular mucosal pattern, light blue crests, and variable vascular density. ${ }^{45,46}$ A Dutch study of 47 patients with GIM who were undergoing surveillance found the sensitivity, specificity positive and negative predictive values to be $71 \%$, 58\%, $65 \%$ and $65 \%$ for NBI, compared to 51\%, 67\%, 62\%, 55\% for white light endoscopy, respectively ${ }^{47}$ Although NBI has decent sensitivity and specificity for the diagnosis of gastric lesions, NBI classification systems vary among studies and suffers from high interobserver variability.

\section{Chromoendoscopy}

Chromoendoscopy relies on dye-based staining of the gastric mucosa with either methylene blue or indigo carmine, which is selectively absorbed by non-acid-producing, mostly absorptive mucosa such as that found in intestinal cells. ${ }^{48}$ This technique can accurately delineate the anatomical extend of minute surface irregularities and histological abnormalities in the stomach, which in turn helps estimate the depth of invasion of early GCs. ${ }^{49}$ It is also useful in determining the lateral borders during endoscopic submucosal dissection. ${ }^{50}$ While chromoendoscopy may help to detect preneoplastic gastric lesions, this technique lengthens the time of the endoscopic procedure, adds to staff workload, and may decrease patient tolerance of the procedure.

\section{Probe-based confocal laser endomicroscopy}

Probe-based confocal laser endomicroscopy (pCLE) provides high-level magnification $(\times 1,000)$ of the gastrointestinal tract epithelium, and has been used for real-time evaluation of gastric lesions. ${ }^{51}$ pCLE requires the intravenous administration of 

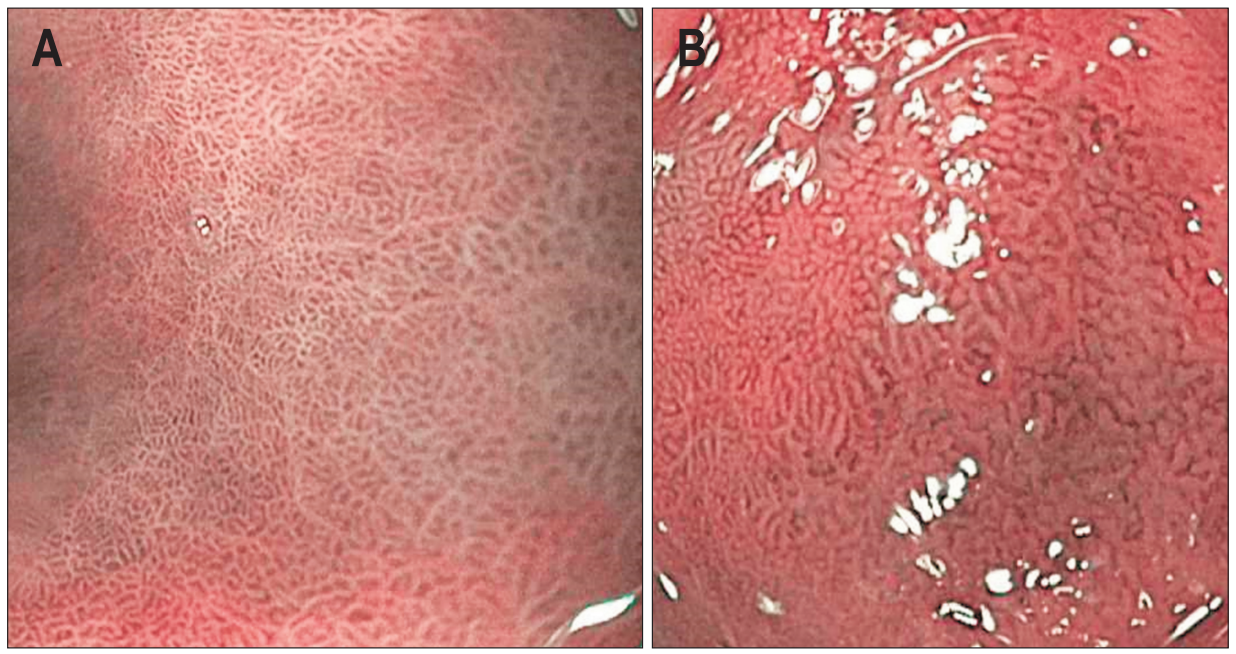

Fig. 4. On magnified narrow-band imaging, the normal gastric mucosa (A) should demonstrate a regular circular pattern and homogeneously spaced gastric pits. In contrast, gastric intestinal metaplasia (B) is characterized by the presence of features such as a tubule-villous mucosal pattern, irregular mucosal pattern, and variable vascular density.

fluorescein, an organic fluorophore which is administered prior to the examination. During pCLE, goblet cells can be clearly identified by the presence of mucin-containing vesicles within the cytoplasm which appear dark due to absence of fluorescein uptake (Fig. 5). pCLE has demonstrated excellent sensitivity and specificity, and additionally provides the benefit of surveying a larger swath of mucosa than is possible to biopsy. ${ }^{52}$ Substitution of a forceps biopsy with an "optical" biopsy may decrease mucosal scar formation which would otherwise confound future lesion determination; pCLE also offers the advantage of realtime diagnosis of lesions, and may therefore permit endoscopic resection during a diagnostic evaluation. Disadvantages of pCLE include the need to stock fluorescein, interobserver variability in interpretation, the high cost of initial capital expenditure, and the operator learning curve required to accurately interpret confocal images.

\section{CURRENT PRACTICES OF ENDOSCOPIC SURVEILLANCE OF GIM}

The optimal practice of surveillance of GIM is currently undefined. The lack of consensus on surveillance is due in part to the heterogeneity in rates of GC not only between different regions of the world, but even heterogeneity within a given country between members of different ethnic or racial groups. In highincidence countries with a relative abundance of resources and a developed endoscopic tradition (such as South Korea and Japan), endoscopic screening for GC of the general population has been proposed. In Japan, biennial screening on a populationlevel with barium meals have been conducted on a prefectural level since $1960 ;^{53}$ in 2016, the Japanese government introduced endoscopic screening as an alternative to barium testing on a national level. ${ }^{54}$ Similarly, as GC is the most common cancer in South Korea, a population-level mass screening program was launched in 2002 consisting of biennial endoscopic examinations for men and women beginning at age $40 .^{53}$ Notably, these

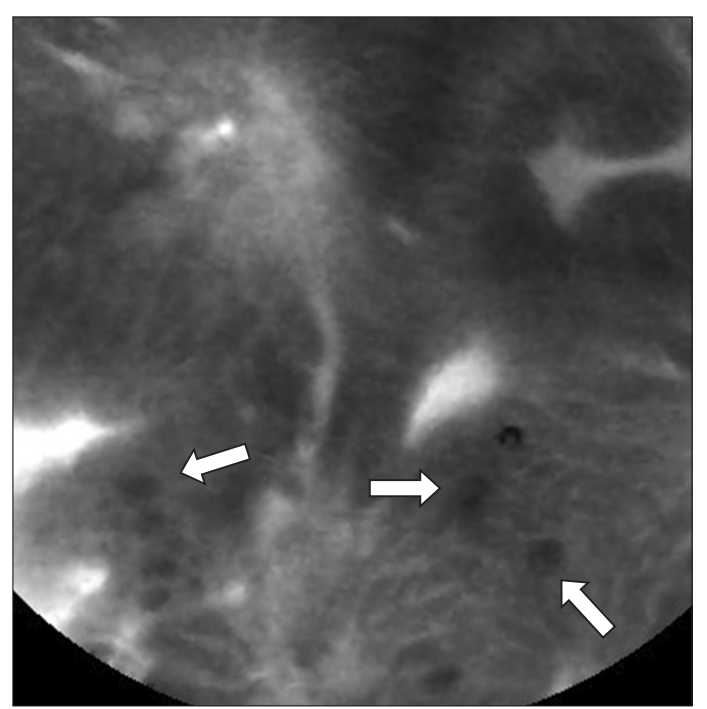

Fig. 5. With probe-based confocal laser endomicroscopy, intestinal cells can clearly be identified by the presence of mucin-containing vesicles within the cytoplasm, which appear dark due to absence of fluorescein uptake (white arrows).

screening recommendations do not make overt reference to surveillance once GIM is diagnosed, but presumably the frequency of surveillance should be at least as intense as that recommended for screening of the general population. A retrospective study from South Korea found that in patients with marked GIM (based upon the Sydney System), a vigilant surveillance strategy of yearly endoscopy led to a higher rate of detection of GC at an early stage compared to usual screening. ${ }^{55}$ Therefore even yearly endoscopies in very high risk individuals may be warranted. While no mass screening programs exist in other regions of high $H$. pylori prevalence and high GC incidence (such as the post-Soviet countries, Latin America, Southeast Asia, and portions of the Middle East), patients from these regions may benefit from GIM surveillance.

In lower-incidence European countries, the European Soci- 
ety for Gastrointestinal Endoscopy (ESGE) recommends that patients with GIM or AG in both antrum and corpus undergo surveillance every 3 years after diagnosis, and patients with mild-to-moderate AG or GIM confined to the antrum not undergo surveillance. ${ }^{42}$ Patients with low-grade dysplasia should be followed-up within a year of diagnosis while those with high-grade dysplasia should be closely followed by endoscopy every 6 months. In the United States, a racially and ethnically heterogeneous nation with a large population of immigrants, no clear guidelines for GIM surveillance exist; however, a recent modeling study suggests that a screening upper endoscopy at the age of 50 and subsequent surveillance if GIM is diagnosed is cost-effective in non-Hispanic black, Hispanic, and Asian Americans. $^{56}$

We recommend a surveillance strategy for the United States which takes into account ethnicity, race, immigration status, and family history (Fig. 6). We recommend that non-Hispanic white Americans and immigrants from low-incidence regions (such as Western Europe or Australia) with biopsy-proven GIM be surveyed with a strategy similar to ESGE guidelines, as antral-only GIM in this population likely carries low risk for progression. African Americans, Asian Americans, Hispanic Americans, immigrants from high-incidence regions, and those with a family history of GC are at much higher risk for GIM progression. As such, in these patients a more intensive surveillance strategy is warranted. Notably, many patients may have GIM diagnosed incidentally on stomach biopsy for other symptoms; therefore, the topographical extent of GIM may not be known. We recommend recalling patients with incidentally diagnosed GIM for a mapping endoscopy, with the interval of recall based on risk group.

\section{CONCLUSIONS}

GIM represents a known premalignant state of the human stomach along the pathway to GC. Prevalence of GIM is highly variable in different regions of the world, and is strongly correlated to both endemicity of $H$. pylori and incidence of GC. While rates of GC are declining worldwide, it remains the third leading cause of cancer-related mortality. GIM can be readily diagnosed by endoscopic biopsy, and with recent advances in endoscopic imaging, potentially optically as well. Thus, endoscopic surveillance of GIM represents an excellent opportunity for early detection and intervention of GC. The United State represents an ethnically and racially diverse nation with a large population of recent immigrants; as such, rates of GIM and GC may be highly variable. We favor a flexible, step-wise approach to surveillance which takes into account known risk factors for GC including race, ethnicity, immigration status, and family history. Clearly, there are still many unanswered questions about the pathogenesis of GIM and its optimal management which are worthy of additional study.

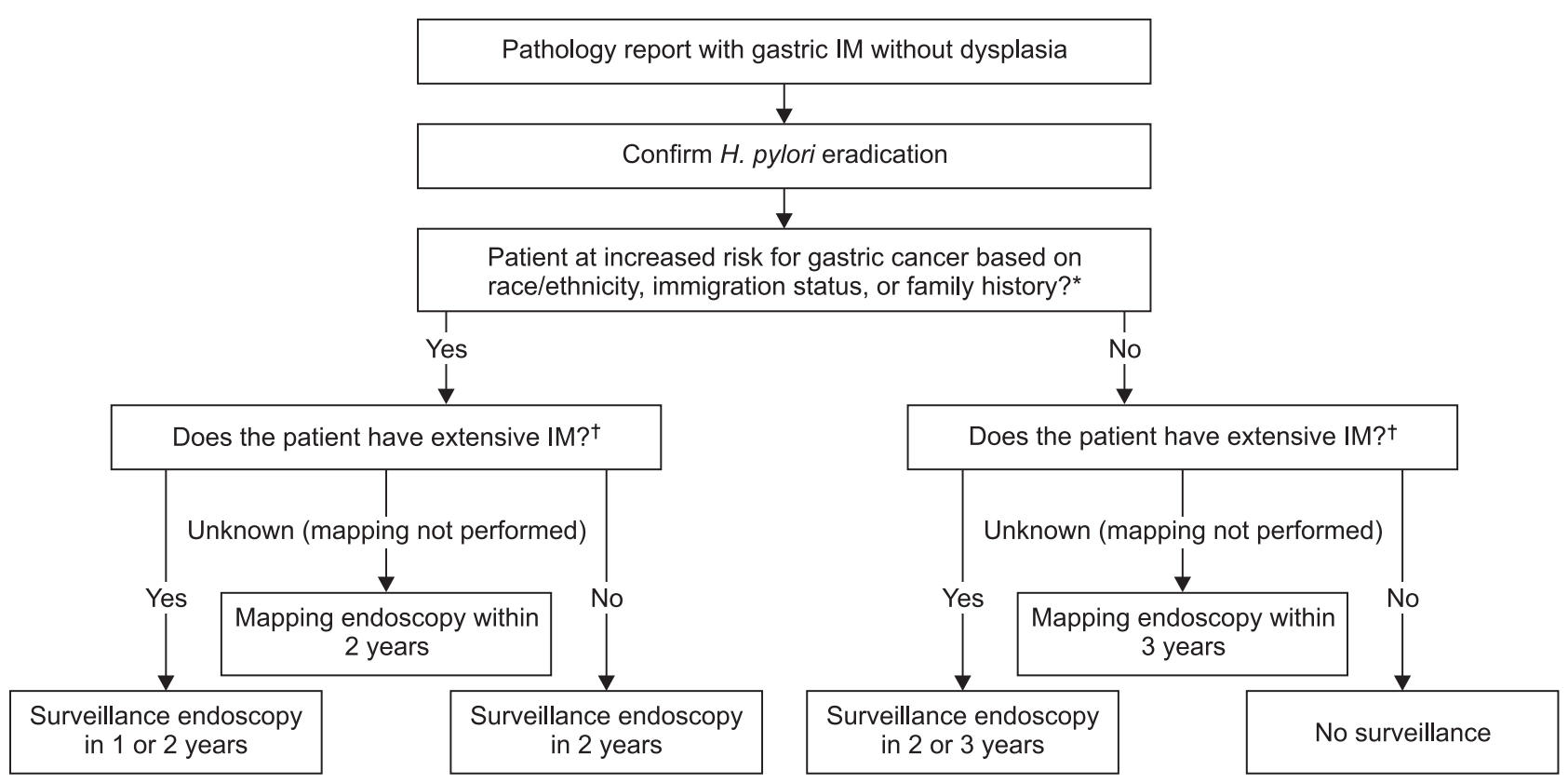

Fig. 6. Recommended surveillance strategy following a gastric biopsy that shows intestinal metaplasia (IM).

$\mathrm{H}$, pylori, Helicobacter pylori. *Increaed risk race/ethinicity defined as African Americans, Asian Americans, and Hispanic Americans of any race. Increased risk immigration status defined as 1st or 2nd (with at least one parent) generation immigrants from high-incidence areas (South America, Central America, Mexico, Caribbean, East Asia, Southeast Asia, post-Soviet states, Iran, and Turcky). Family history defined as at least one first-degree relative with gastric cancer; ${ }^{\dagger}$ Based upon biopsies from antrum and corpus, and from lesser and greater curvatures, in separate bottles. Extensive IM involving both antrum and corpus. 


\section{CONFLICTS OF INTEREST}

No potential conflict of interest relevant to this article was reported.

\section{ORCID}

Robert J. Huang

Alyssa Y. Choi

Joo Ha Hwang https://orcid.org/0000-0002-0891-7409 https://orcid.org/0000-0002-6830-9384 https://orcid.org/0000-0002-7534-230X

\section{REFERENCES}

1. Park YH, Kim N. Review of atrophic gastritis and intestinal metaplasia as a premalignant lesion of gastric cancer. J Cancer Prev 2015;20:25-40.

2. ASGE Standards of Practice Committee, Evans JA, Chandrasekhara V, et al. The role of endoscopy in the management of premalignant and malignant conditions of the stomach. Gastrointest Endosc 2015;82:1-8.

3. Kim SG, Jung HK, Lee HL, et al. Guidelines for the diagnosis and treatment of Helicobacter pylori infection in Korea, 2013 revised edition. J Gastroenterol Hepatol 2014;29:1371-1386.

4. Leung WK, Sung JJ. Review article: intestinal metaplasia and gastric carcinogenesis. Aliment Pharmacol Ther 2002;16:1209-1216.

5. Uemura N, Okamoto S, Yamamoto S, et al. Helicobacter pylori infection and the development of gastric cancer. N Engl J Med 2001;345:784-789.

6. Sipponen P, Kimura K. Intestinal metaplasia, atrophic gastritis and stomach cancer: trends over time. Eur J Gastroenterol Hepatol 1994;6:S79-S83.

7. Lim JH, Kim N, Lee HS, et al. Correlation between endoscopic and histological diagnoses of gastric intestinal metaplasia. Gut Liver 2013;7:41-50.

8. Marques-Silva L, Areia M, Elvas L, Dinis-Ribeiro M. Prevalence of gastric precancerous conditions: a systematic review and metaanalysis. Eur J Gastroenterol Hepatol 2014;26:378-387.

9. Fennerty MB. Gastric intestinal metaplasia on routine endoscopic biopsy. Gastroenterology 2003;125:586-590.

10. Song H, Ekheden IG, Zheng Z, Ericsson J, Nyrén O, Ye W. Incidence of gastric cancer among patients with gastric precancerous lesions: observational cohort study in a low risk Western population. BMJ 2015;351:h3867.

11. Torre LA, Bray F, Siegel RL, Ferlay J, Lortet-Tieulent J, Jemal A. Global cancer statistics, 2012. CA Cancer J Clin 2015;65:87-108.

12. Correa P. Human gastric carcinogenesis: a multistep and multifactorial process. First American Cancer Society Award Lecture on Cancer Epidemiology and Prevention. Cancer Res 1992;52:67356740.

13. Ishaq S, Nunn L. Helicobacter pylori and gastric cancer: a state of the art review. Gastroenterol Hepatol Bed Bench 2015;8:S6-S14.

14. Goh KL, Chan WK, Shiota S, Yamaoka Y. Epidemiology of Helico- bacter pylori infection and public health implications. Helicobacter 2011;16:1-9.

15. Eslick GD, Lim LL, Byles JE, Xia HH, Talley NJ. Association of Helicobacter pylori infection with gastric carcinoma: a meta-analysis. Am J Gastroenterol 1999;94:2373-2379.

16. Peleteiro B, Bastos A, Ferro A, Lunet N. Prevalence of Helicobacter pylori infection worldwide: a systematic review of studies with national coverage. Dig Dis Sci 2014;59:1698-1709.

17. Li N, Xie C, Lu NH. Transforming growth factor-beta: an important mediator in Helicobacter pylori-associated pathogenesis. Front Cell Infect Microbiol 2015;5:77.

18. Sue S, Shibata W, Maeda S. Helicobacter pylori-induced signaling pathways contribute to intestinal metaplasia and gastric carcinogenesis. Biomed Res Int 2015;2015:737621.

19. Valenzuela MA, Canales J, Corvalán AH, Quest AF. Helicobacter pylori-induced inflammation and epigenetic changes during gastric carcinogenesis. World J Gastroenterol 2015;21:12742-12756.

20. Ford AC, Forman D, Hunt R, Yuan Y, Moayyedi P. Helicobacter pylori eradication for the prevention of gastric neoplasia. Cochrane Database Syst Rev 2015;(7):CD005583.

21. Ford AC, Forman D, Hunt RH, Yuan Y, Moayyedi P. Helicobacter pylori eradication therapy to prevent gastric cancer in healthy asymptomatic infected individuals: systematic review and metaanalysis of randomised controlled trials. BMJ 2014;348:g3174.

22. Asaka M, Kato M, Takahashi S, et al. Guidelines for the management of Helicobacter pylori infection in Japan: 2009 revised edition. Helicobacter 2010;15:1-20.

23. Yeh JM, Kuntz KM, Ezzati M, Goldie SJ. Exploring the costeffectiveness of Helicobacter pylori screening to prevent gastric cancer in China in anticipation of clinical trial results. Int J Cancer 2009;124:157-166.

24. Rugge M, Capelle LG, Cappellesso R, Nitti D, Kuipers EJ. Precancerous lesions in the stomach: from biology to clinical patient management. Best Pract Res Clin Gastroenterol 2013;27:205-223.

25. Ahn HJ, Lee DS. Helicobacter pylori in gastric carcinogenesis. World J Gastrointest Oncol 2015;7:455-465.

26. Zhu H, Yang X, Zhang C, et al. Red and processed meat intake is associated with higher gastric cancer risk: a meta-analysis of epidemiological observational studies. PLoS One 2013;8:e70955.

27. Zhong C, Li KN, Bi JW, Wang BC. Sodium intake, salt taste and gastric cancer risk according to Helicobacter pylori infection, smoking, histological type and tumor site in China. Asian Pac J Cancer Prev 2012;13:2481-2484.

28. Ladeiras-Lopes R, Pereira AK, Nogueira A, et al. Smoking and gastric cancer: systematic review and meta-analysis of cohort studies. Cancer Causes Control 2008;19:689-701.

29. González CA, Pera G, Agudo A, et al. Smoking and the risk of gastric cancer in the European Prospective Investigation Into Cancer and Nutrition (EPIC). Int J Cancer 2003;107:629-634.

30. Turati F, Tramacere I, La Vecchia C, Negri E. A meta-analysis of body mass index and esophageal and gastric cardia adenocarcinoma. Ann Oncol 2013;24:609-617. 
31. Yang P, Zhou Y, Chen B, et al. Overweight, obesity and gastric cancer risk: results from a meta-analysis of cohort studies. Eur J Cancer 2009;45:2867-2873.

32. Dixon MF, Genta RM, Yardley JH, Correa P. Classification and grading of gastritis: the updated Sydney System. International Workshop on the Histopathology of Gastritis, Houston 1994. Am J Surg Pathol 1996;20:1161-1181.

33. Rugge M, Meggio A, Pennelli G, et al. Gastritis staging in clinical practice: the OLGA staging system. Gut 2007;56:631-636.

34. Rugge M, de Boni M, Pennelli G, et al. Gastritis OLGA-staging and gastric cancer risk: a twelve-year clinico-pathological follow-up study. Aliment Pharmacol Ther 2010;31:1104-1111.

35. Capelle LG, de Vries AC, Haringsma J, et al. The staging of gastritis with the OLGA system by using intestinal metaplasia as an accurate alternative for atrophic gastritis. Gastrointest Endosc 2010;71:1150-1158.

36. Filipe MI, Potet F, Bogomoletz WV, et al. Incomplete sulphomucinsecreting intestinal metaplasia for gastric cancer. Preliminary data from a prospective study from three centres. Gut 1985;26:13191326.

37. Filipe MI, Muñoz N, Matko I, et al. Intestinal metaplasia types and the risk of gastric cancer: a cohort study in Slovenia. Int J Cancer 1994;57:324-329.

38. Piazuelo MB, Haque S, Delgado A, Du JX, Rodriguez F, Correa P. Phenotypic differences between esophageal and gastric intestinal metaplasia. Mod Pathol 2004;17:62-74.

39. Mirza ZK, Das KK, Slate J, et al. Gastric intestinal metaplasia as detected by a monoclonal antibody is highly associated with gastric adenocarcinoma. Gut 2003;52:807-812.

40. Tanaka H, Tsukamoto T, Mizoshita T, et al. Expression of small intestinal and colonic phenotypes in complete intestinal metaplasia of the human stomach. Virchows Arch 2005;447:806-815.

41. Schlemper RJ, Riddell RH, Kato Y, et al. The Vienna classification of gastrointestinal epithelial neoplasia. Gut 2000;47:251-255.

42. Dinis-Ribeiro M, Areia M, de Vries AC, et al. Management of precancerous conditions and lesions in the stomach (MAPS): guideline from the European Society of Gastrointestinal Endoscopy (ESGE), European Helicobacter Study Group (EHSG), European Society of Pathology (ESP), and the Sociedade Portuguesa de Endoscopia Digestiva (SPED). Virchows Arch 2012;460:19-46.

43. Kikuste I, Marques-Pereira R, Monteiro-Soares M, et al. Systematic review of the diagnosis of gastric premalignant conditions and neoplasia with high-resolution endoscopic technologies. Scand J Gastroenterol 2013;48:1108-1117.

44. Osawa H, Yamamoto H. Present and future status of flexible spectral imaging color enhancement and blue laser imaging technology. Dig Endosc 2014;26:105-115.

45. Pimentel-Nunes P, Dinis-Ribeiro M, Soares JB, et al. A multicenter validation of an endoscopic classification with narrow band imaging for gastric precancerous and cancerous lesions. Endoscopy 2012;44:236-246.

46. Pimentel-Nunes P, Libânio D, Lage J, et al. A multicenter prospective study of the real-time use of narrow-band imaging in the diagnosis of premalignant gastric conditions and lesions. Endoscopy 2016;48:723-730.

47. Capelle LG, Haringsma J, de Vries AC, et al. Narrow band imaging for the detection of gastric intestinal metaplasia and dysplasia during surveillance endoscopy. Dig Dis Sci 2010;55:3442-3448.

48. Taghavi SA, Membari ME, Eshraghian A, Dehghani SM, Hamidpour L, Khademalhoseini F. Comparison of chromoendoscopy and conventional endoscopy in the detection of premalignant gastric lesions. Can J Gastroenterol 2009;23:105-108.

49. Dinis-Ribeiro M. Chromoendoscopy for early diagnosis of gastric cancer. Eur J Gastroenterol Hepatol 2006;18:831-838.

50. Lee JH, Kim JG, Jung HK, et al. Clinical practice guidelines for gastric cancer in Korea: an evidence-based approach. J Gastric Cancer 2014;14:87-104.

51. Ballester V, Cruz-Correa M. Endoscopic surveillance of gastrointestinal premalignant lesions: current knowledge and future directions. Curr Opin Gastroenterol 2014;30:477-483.

52. Templeton A, Hwang JH. Confocal microscopy in the esophagus and stomach. Clin Endosc 2013;46:445-449.

53. Leung WK, Wu MS, Kakugawa Y, et al. Screening for gastric cancer in Asia: current evidence and practice. Lancet Oncol 2008;9:279-287.

54. Hamashima C, Goto R. Potential capacity of endoscopic screening for gastric cancer in Japan. Cancer Sci 2017;108:101-107.

55. Yoon H, Kim N, Lee HS, et al. Effect of endoscopic screening at 1-year intervals on the clinicopathologic characteristics and treatment of gastric cancer in South Korea. J Gastroenterol Hepatol 2012;27:928-934.

56. Saumoy M, Schneider Y, Shen N, Kahaleh M, Sharaiha RZ, Shah SC. Cost effectiveness of gastric cancer screening according to race and ethnicity. Gastroenterology 2018;155:648-660. 